PROCEEDINGS OF THE

AMERICAN MATHEMATICAL SOCIETY

Volume 134, Number 6, Pages 1719-1725

S 0002-9939(05)08173-6

Article electronically published on December 5, 2005

\title{
SEMI-FINITE FORMS OF BILATERAL BASIC HYPERGEOMETRIC SERIES
}

\author{
WILLIAM Y. C. CHEN AND AMY M. FU
}

(Communicated by John R. Stembridge)

\begin{abstract}
We show that several classical bilateral summation and transformation formulas have semi-finite forms. We obtain these semi-finite forms from unilateral summation and transformation formulas. Our method can be applied to derive Ramanujan's ${ }_{1} \psi_{1}$ summation, Bailey's ${ }_{2} \psi_{2}$ transformations, and Bailey's ${ }_{6} \psi_{6}$ summation.
\end{abstract}

\section{INTRODUCTION}

We follow the terminology for basic hypergeometric series in [6]. Assuming $|q|<1$, let

$$
(a ; q)_{\infty}=(1-a)(1-a q)\left(1-a q^{2}\right) \cdots .
$$

For any integer $n$, the $q$-shifted factorial $(a ; q)_{n}$ is given by

$$
(a ; q)_{n}=\frac{(a ; q)_{\infty}}{\left(a q^{n} ; q\right)_{\infty}} .
$$

For $n \geq 0$, we have the following relation which is crucial for this paper:

$$
(a ; q)_{-n}=\frac{1}{\left(a q^{-n} ; q\right)_{n}}=\frac{(-q / a)^{n} q^{\left(\begin{array}{c}
n \\
2
\end{array}\right)}}{(q / a ; q)_{n}} .
$$

For convenience, we employ the following usual notation:

$$
\left(a_{1}, a_{2}, \cdots, a_{m} ; q\right)_{n}=\left(a_{1} ; q\right)_{n}\left(a_{2} ; q\right)_{n} \cdots\left(a_{m} ; q\right)_{n} .
$$

The (unilateral) basic hypergeometric series ${ }_{r+1} \phi_{r}$ is defined by

$$
{ }_{r+1} \phi_{r}\left[\begin{array}{l}
a_{1}, a_{2}, \cdots, a_{r+1} \\
b_{1}, b_{2}, \cdots, b_{r}
\end{array} ; q, z\right]=\sum_{k=0}^{\infty} A(k),
$$

where

$$
A(k)=\frac{\left(a_{1}, a_{2}, \cdots, a_{r+1} ; q\right)_{k}}{\left(b_{1}, b_{2}, \cdots, b_{r}, q ; q\right)_{k}} z^{k} .
$$

The bilateral basic hypergeometric series ${ }_{s} \psi_{s}$ is defined as follows:

$$
{ }_{s} \psi_{s}\left[\begin{array}{l}
a_{1}, a_{2}, \cdots, a_{s} \\
b_{1}, b_{2}, \cdots, b_{s}
\end{array} ; q, z\right]=\sum_{k=-\infty}^{\infty} B(k)
$$

Received by the editors December 8, 2004 and, in revised form, January 11, 2005.

2000 Mathematics Subject Classification. Primary 33D15.

Key words and phrases. Bilateral hypergeometric summation, semi-finite forms, Ramanujan's ${ }_{1} \psi_{1}$ summation, Bailey's ${ }_{2} \psi_{2}$ transformations, Bailey's ${ }_{6} \psi_{6}$ summation. 
where

$$
B(k)=\frac{\left(a_{1}, a_{2}, \cdots, a_{s} ; q\right)_{k}}{\left(b_{1}, b_{2}, \cdots, b_{s} ; q\right)_{k}} z^{k} .
$$

In this paper, we propose the following method for deriving bilateral summation and transformation formulas using semi-finite forms. For a bilateral series ${ }_{s} \psi_{s}$ as given in (1.3), we construct a summand $G(k, m)$ which implies a unilateral series ${ }_{r+s+1} \phi_{r+s}$, where $r$ is a nonnegative integer, such that

$$
\lim _{m \rightarrow \infty} G(k, m)=B(k)
$$

for all $k$, and the summation

$$
\sum_{k=-m}^{\infty} G(k, m)
$$

can be easily accomplished as a Laurent extension of the summation

$$
\sum_{k=0}^{\infty} G(k-m, m)=G(-m, m) \sum_{k=0}^{\infty} A(k),
$$

where $G(k, m)$ can be written as

$$
G(k-m, m)=G(-m, m) A(k)
$$

for some $A(k)$. The bilateral series (1.3) is then obtained from (1.4) as $m \rightarrow \infty$, subject to suitable convergence conditions. We apply this procedure to derive bilateral series identities from suitable unilateral ones. The above summation (1.4) is called the semi-finite form of the bilateral summation (1.3). A method similar to ours was recently used by Schlosser [9], and Jouhet and Schlosser [8, who derived summations for bilateral series from finite forms. We also note that another method, which uses a similar factorization as above, for deriving bilateral series identities from unilateral ones was used by Ismail [7, and Askey and Ismail 2. Rather than taking limits, they apply analytic continuation as the main ingredient.

In this paper, we present semi-finite forms of several classical bilateral summation and transformation formulas such as Ramanujan's ${ }_{1} \psi_{1}$ summation, Bailey's ${ }_{2} \psi_{2}$ transformations, and Bailey's ${ }_{6} \psi_{6}$ summation.

\section{FrOM $_{2} \phi_{1} \mathrm{TO}_{1} \psi_{1}$}

Using the well-known Gauss summation formula

$$
{ }_{2} \phi_{1}\left[\begin{array}{c}
a, b \\
{ }_{c}
\end{array} q, c / a b\right]=\frac{(c / a, c / b ; q)_{\infty}}{(c, c / a b ; q)_{\infty}},
$$

where $|c / a b|<1$, we get a semi-finite form of Ramanujan's summation of the general ${ }_{1} \psi_{1}$,

$$
{ }_{1} \psi_{1}\left[\begin{array}{l}
a \\
{ }_{b}
\end{array} q, z\right]=\sum_{k=-\infty}^{\infty} \frac{(a ; q)_{k}}{(b ; q)_{k}} z^{k}=\frac{(q ; q)_{\infty}(b / a ; q)_{\infty}(a z ; q)_{\infty}(q / a z ; q)_{\infty}}{(b ; q)_{\infty}(q / a ; q)_{\infty}(z ; q)_{\infty}(b / a z ; q)_{\infty}}
$$

where $|b / a|<|z|<1$.

Proposition 2.1. For $|z|<1$, the following identity holds:

$$
\sum_{k=-m}^{\infty} \frac{(a ; q)_{k}\left(b q^{m} / a z ; q\right)_{k}}{\left(q^{1+m} ; q\right)_{k}(b ; q)_{k}} z^{k}=\frac{(q ; q)_{m}(q / a z ; q)_{m}}{(q / a ; q)_{m}(b / a z ; q)_{m}} \frac{(b / a ; q)_{\infty}(a z ; q)_{\infty}}{(b ; q)_{\infty}(z ; q)_{\infty}} .
$$


Proof. The left-hand side of (2.3) can be rewritten as

$$
\begin{aligned}
& \sum_{k=0}^{\infty} \frac{(a ; q)_{k-m}\left(b q^{m} / a z ; q\right)_{k-m}}{\left(q^{1+m} ; q\right)_{k-m}(b ; q)_{k-m}} z^{k-m} \\
& =z^{-m} \frac{(a ; q)_{-m}\left(b q^{m} / a z ; q\right)_{-m}}{\left(q^{1+m} ; q\right)_{-m}(b ; q)_{-m}} \sum_{k=0}^{\infty} \frac{\left(a q^{-m} ; q\right)_{k}(b / a z ; q)_{k}}{(q ; q)_{k}\left(b q^{-m} ; q\right)_{k}} z^{k} \\
& \stackrel{(2.1)}{=} z^{-m} \frac{(a ; q)_{-m}\left(b q^{m} / a z ; q\right)_{-m}}{\left(q^{1+m} ; q\right)_{-m}(b ; q)_{-m}} \frac{(b / a ; q)_{\infty}\left(a z q^{-m} ; q\right)_{\infty}}{\left(b q^{-m} ; q\right)_{\infty}(z ; q)_{\infty}} \\
& \stackrel{(1.1)}{=} z^{-m} \frac{(q ; q)_{m}\left(a z q^{-m} ; q\right)_{m}}{\left(a q^{-m} ; q\right)_{m}(b / a z ; q)_{m}} \frac{(a z ; q)_{\infty}(b / a ; q)_{\infty}}{(b ; q)_{\infty}(z ; q)_{\infty}}
\end{aligned}
$$

which equals the right-hand side of (2.3).

Taking the limit $m \rightarrow \infty$ in Proposition 2.1 while assuming $|b / a z|<1$, we immediately obtain (2.2).

We remark that our method is different from the method of M. Jackson's elementary proof of (2.2) (see the exposition of Schlosser 9]) in the sense that Jackson's proof does not give a semi-finite form although the Gauss summation is also the basic ingredient. We should also note that a finite form of Ramanujan's ${ }_{1} \psi_{1}$ summation has been given by Schlosser [10] using the terminating $q$-Pfaff-Saalschütz summation.

\section{FrOM ${ }_{3} \phi_{2}$ TO ${ }_{2} \psi_{2}$}

In this section, we use two ${ }_{3} \phi_{2}$ summation and transformation formulas to give the semi-finite forms of ${ }_{2} \psi_{2}$ formulas due to Bailey. We begin with the following ${ }_{2} \psi_{2}$ transformation formula [6, Ex. 5.20(i)] valid for $|z|,|c d / a b z|,|d / a|,|c / b|<1$,

$$
{ }_{2} \psi_{2}\left[\begin{array}{l}
a, b \\
c, d
\end{array} ; q, z\right]=\frac{(a z, d / a, c / b, d q / a b z ; q)_{\infty}}{(z, d, q / b, c d / a b z ; q)_{\infty}}{ }_{2} \psi_{2}\left[\begin{array}{l}
a, a b z / d \\
a z, c
\end{array} ; q, \frac{d}{a}\right] .
$$

Using a $q$-analogue of the Kummer-Thomae-Whipple formula [6, Eq. (3.2.7)],

$$
{ }_{3} \phi_{2}\left[\begin{array}{l}
a, b, c \\
d, e
\end{array} ; q, \frac{d e}{a b c}\right]=\frac{(e / a, d e / b c ; q)_{\infty}}{(e, d e / a b c ; q)_{\infty}} 3 \phi_{2}\left[\begin{array}{l}
a, d / b, d / c \\
d, d e / b c
\end{array} ; q, \frac{e}{a}\right],
$$

where $|d e / a b c|<1$ and $|e / a|<1$, we get a semi-finite form of (3.1).

Proposition 3.1. For $|z|<1$ and $|d / a|<1$, we have

$$
\begin{aligned}
\sum_{k=-m}^{\infty} \frac{(a, b ; q)_{k}\left(c d q^{m} / a b z ; q\right)_{k}}{(c, d ; q)_{k}\left(q^{1+m} ; q\right)_{k}} z^{k}= & \frac{(a z, d / a ; q)_{\infty}}{(z, d ; q)_{\infty}} \frac{(c / b, d q / a b z ; q)_{m}}{(q / b, c d / a b z ; q)_{m}} \\
& \cdot \sum_{k=-m}^{\infty} \frac{\left(a, c q^{m} / b, a b z / d ; q\right)_{k}}{\left(c, q^{1+m}, a z ; q\right)_{k}}(d / a)^{k}
\end{aligned}
$$


Proof. The left-hand side of (3.3) equals

$$
\begin{gathered}
z^{-m} \frac{\left(a, b, c d q^{m} / a b z ; q\right)_{-m}}{\left(c, d, q^{1+m} ; q\right)_{-m}} \sum_{k=0}^{\infty} \frac{\left(a q^{-m}, b q^{-m}, c d / a b z ; q\right)_{k}}{\left(c q^{-m}, d q^{-m}, q ; q\right)_{k}} z^{k} \\
\stackrel{(3.2)}{=} z^{-m} \frac{\left(a, b, c d q^{m} / a b z ; q\right)_{-m}}{\left(c, d, q^{1+m} ; q\right)_{-m}} \frac{\left(d / a, a z q^{-m} ; q\right)_{\infty}}{\left(d q^{-m}, z ; q\right)_{\infty}} \\
\stackrel{\sum_{k=0}^{\infty}}{\stackrel{\left(a q^{-m}, c / b, a b z q^{-m} / d ; q\right)_{k}}{\left(q, c q^{-m}, a z q^{-m} ; q\right)_{k}}\left(\frac{d}{a}\right)^{k}} \frac{(d / a, a z ; q)_{\infty}}{(d, z ; q)_{\infty}} \frac{\left(c / b, a b z q^{-m} / d ; q\right)_{m}}{\left(b q^{-m}, c d / a b z ; q\right)_{m}}\left(\frac{d}{a z}\right)^{m} \\
\cdot \sum_{k=0}^{\infty} \frac{\left(a, c q^{m} / b, a b z / d ; q\right)_{k-m}}{\left(c, q^{1+m}, a z ; q\right)_{k-m}}(d / a)^{k-m}
\end{gathered}
$$

which can be rewritten in the form of the right-hand side of (3.3).

The next ${ }_{2} \psi_{2}$ transformation formula we consider is the following [6, Ex. 5.20(ii)]:

$$
{ }_{2} \psi_{2}\left[\begin{array}{l}
a, b \\
c, d
\end{array} ; q, z\right]=\frac{(a z, b z, c q / a b z, d q / a b z ; q)_{\infty}}{(q / a, q / b, c, d ; q)_{\infty}} \psi_{2}\left[\begin{array}{l}
a b z / c, a b z / d \\
a z, b z ; q, \frac{c d}{a b z}
\end{array}\right]
$$

Using a summation of Hall [6, Eq. (3.2.10)],

$$
{ }_{3} \phi_{2}\left[\begin{array}{l}
a, b, c \\
d, e
\end{array} ; q, \frac{d e}{a b c}\right]=\frac{(b, d e / a b, d e / b c ; q)_{\infty}}{(d, e, d e / a b c ; q)_{\infty}}{ }_{3} \phi_{2}\left[\begin{array}{l}
d / b, e / b, d e / a b c \\
d e / a b, d e / b c ; q, b
\end{array}\right]
$$

where $|d e / a b c|<1$ and $|b|<1$, we obtain the following semi-finite form of (3.4).

Proposition 3.2. For $|z|<1$ and $|c d / a b z|<1$, we have

$$
\begin{aligned}
\sum_{k=-m}^{\infty} \frac{(a, b ; q)_{k}\left(c d q^{m} / a b z ; q\right)_{k}}{(c, d ; q)_{k}\left(q^{1+m} ; q\right)_{k}} z^{k}= & \frac{(a z, b z, c d / a b z ; q)_{\infty}}{(c, d, z ; q)_{\infty}} \frac{(c q / a b z, d q / a b z, z ; q)_{m}}{(q / a, q / b, c d / a b z ; q)_{m}} \\
& \cdot \sum_{k=-m}^{\infty} \frac{\left(a b z / c, a b z / d, z q^{m} ; q\right)_{k}}{\left(a z, b z, q^{1+m} ; q\right)_{k}}(c d / a b z)^{k}
\end{aligned}
$$

\section{From nOnterminating ${ }_{8} \phi_{7}$ TO ${ }_{6} \psi_{6}$}

In this section, we give a semi-finite form of Bailey's ${ }_{6} \psi_{6}$ summation formula [4] by using Bailey's 3-term transformation formula for a nonterminating, very well- 
poised ${ }_{8} \phi_{7}$ series [6, Eq. (2.11.1)]:

$$
\begin{aligned}
& { }_{8} \phi_{7}\left[\begin{array}{l}
a, q a^{\frac{1}{2}},-q a^{\frac{1}{2}}, b, c, d, e, f \\
a^{\frac{1}{2}},-a^{\frac{1}{2}}, a q / b, a q / c, a q / d, a q / e, a q / f
\end{array} ; q, \frac{a^{2} q^{2}}{b c d e f}\right] \\
& =\frac{(a q, a q / d e, a q / d f, a q / e f, e q / c, f q / c, b / a, b e f / a ; q)_{\infty}}{(a q / d, a q / e, a q / f, a q / d e f, q / c, e f q / c, b e / a, b f / a ; q)_{\infty}} \\
& { }_{8} \phi_{7}\left[\begin{array}{c}
e f / c, q(e f / c)^{\frac{1}{2}},-q(e f / c)^{\frac{1}{2}}, a q / b c, a q / c d, e f / a, e, f \\
(e f / c)^{\frac{1}{2}},-(e f / c)^{\frac{1}{2}}, b e f / a, d e f / a, a q / c, f q / c, e q / c
\end{array} ; q, \frac{b d}{a}\right] \\
& +\frac{b}{a} \frac{(a q, b q / a, b q / c, b q / d, b q / e, b q / f, d, e, f ; q)_{\infty}}{(a q / b, a q / c, a q / d, a q / e, a q / f, b d / a, b e / a, b f / a, d e f / a ; q)_{\infty}} \\
& \frac{\left(a q / b c, b d e f / a^{2}, a^{2} q / b d e f ; q\right)_{\infty}}{\left(a q / \operatorname{def}, q / c, b^{2} q / a ; q\right)_{\infty}} \\
& \cdot{ }_{8} \phi_{7}\left[\begin{array}{l}
b^{2} / a, q b a^{-\frac{1}{2}},-q b a^{-\frac{1}{2}}, b, b c / a, b d / a, b e / a, b f / a \\
b a^{-\frac{1}{2}},-b a^{-\frac{1}{2}}, b q / a, b q / c, b q / d, b q / e, b q / f
\end{array} ;, \frac{a^{2} q^{2}}{b c d e f}\right],
\end{aligned}
$$

where $|b d / a|<1$ and $\left|a^{2} q^{2} / b c d e f\right|<1$.

Proposition 4.1. When $|b d / a|<1$ and $\left|a^{2} q^{2} / b c d e f\right|<1$, we have

$$
\begin{aligned}
\sum_{k=-m}^{\infty} \frac{\left(q^{m-k+1} / a, f q^{m} ; q\right)_{k}}{\left(q^{m-k} f / a, q^{1+m} ; q\right)_{k}} \frac{\left(q a^{\frac{1}{2}},-q a^{\frac{1}{2}}, b, c, d, e ; q\right)_{k}}{\left(a^{\frac{1}{2}},-a^{\frac{1}{2}}, a q / b, a q / c, a q / d, a q / e ; q\right)_{k}}\left(\frac{q a^{2}}{b c d e}\right)^{k} \\
=\frac{1-e f q^{2 m} / c}{1-e f q^{m} / c} \frac{\left(q / a, d f / a, e f / a, a q / b c, a q / c d, e f q^{m} / a ; q\right)_{m}}{\left(f / a, q / b, q / c, q / d, d e f / a, f q^{1+m} / c ; q\right)_{m}} \\
\quad \times \frac{\left(a q, a q / d e, a q / d f, a q / e f, e q^{1+m} / c, f q^{1+m} / c, b / a, b e f q^{m} / a ; q\right)_{\infty}}{\left(a q / d, a q / e, a q / f, a q / d e f, q^{1+m} / c, e f q^{1+m} / c, b e / a, b f q^{m} / a ; q\right)_{\infty}} \\
\quad \sum_{k=-m}^{\infty} \frac{\left(e f q^{m} / c, q^{1+m}(e f / c)^{\frac{1}{2}},-q^{1+m}(e f / c)^{\frac{1}{2}}, a q^{1+m} / b c ; q\right)_{k}}{\left(q^{1+m}, q^{m}(e f / c)^{\frac{1}{2}},-q^{m}(e f / c)^{\frac{1}{2}}, b e f q^{m} / a ; q\right)_{k}} \\
\quad \cdot \frac{\left(a q^{1+m} / c d, e f q^{2 m} / a, e, f q^{m} ; q\right)_{k}}{\left(d e f q^{m} / a, a q / c, f q^{1+2 m} / c, e q^{1+m} / c ; q\right)_{k}}\left(\frac{b d}{a}\right)^{k} \\
+\frac{b}{a} \frac{1-b^{2} q^{2 m} / a}{1-b^{2} q^{m} / a}\left(\frac{a^{2} q}{b c d e}\right)^{m} \frac{(q / a, b c / a ; q)_{m}}{(f / a ; q)_{m}} \frac{\left(a q, b q^{1+2 m} / a, b q^{1+m} / c ; q\right)_{\infty}}{(a q / b, a q / c, a q / d ; q)_{\infty}} \\
\quad \times \frac{\left(b q^{1+m} / d, b q^{1+m} / e, b q / f, d, e, f q^{m}, a q / b c, b d e f / a^{2}, a^{2} q / b d e f ; q\right)_{\infty}}{\left(a q / e, a q / f, b d q^{m} / a, b e q^{m} / a, b f q^{2 m} / a, d e f / a, a q / d e f, q / c, b^{2} q^{1+m} / a ; q\right)_{\infty}} \\
\quad \times \sum_{k=-m}^{\infty} \frac{\left(b^{2} q^{m} / a, q^{1+m} b a^{-\frac{1}{2}},-q^{1+m} b a^{-\frac{1}{2}} ; q\right)_{k}}{\left(q^{1+m}, q^{m} b a^{-\frac{1}{2}},-q^{m} b a^{-\frac{1}{2}} ; q\right)_{k}} \\
\quad \cdot \frac{\left(b, b c q^{m} / a, b d q^{m} / a, b e q^{m} / a, b f q^{2 m} / a ; q\right)_{k}}{\left(b q^{1+2 m} / a, b q^{1+m} / c, b q^{1+m} / d, b q^{1+m} / e, b q / f ; q\right)_{k}}\left(\frac{a^{2} q^{2}}{b c d e f}\right)^{k} \cdot
\end{aligned}
$$


Proof. The left-hand side of (4.2) equals

$$
\begin{aligned}
& \sum_{k=-m}^{\infty} \frac{\left(a q^{-m}, f q^{m} ; q\right)_{k}}{\left(a q^{1-m} / f, q^{1+m} ; q\right)_{k}} \frac{\left(q a^{\frac{1}{2}},-q a^{\frac{1}{2}}, b, c, d, e ; q\right)_{k}}{\left(a^{\frac{1}{2}},-a^{\frac{1}{2}}, a q / b, a q / c, a q / d, a q / e ; q\right)_{k}}\left(\frac{q^{2} a^{2}}{b c d e f}\right)^{k} \\
& \stackrel{(1.5)}{=} \frac{\left(a q^{-m}, f q^{m}, q a^{\frac{1}{2}},-q a^{\frac{1}{2}}, b, c, d, e ; q\right)_{-m}}{\left(a q^{1-m} / f, q^{1+m}, a^{\frac{1}{2}},-a^{\frac{1}{2}}, a q / b, a q / c, a q / d, a q / e ; q\right)_{-m}}\left(\frac{q^{2} a^{2}}{b c d e f}\right)^{-m} \\
& \times \sum_{k=0}^{\infty} \frac{\left(a q^{-2 m}, q^{1-m} a^{\frac{1}{2}},-q^{1-m} a^{\frac{1}{2}} ; q\right)_{k}}{\left(q, q^{-m} a^{\frac{1}{2}},-q^{-m} a^{\frac{1}{2}} ; q\right)_{k}} \\
& \cdot \frac{\left(b q^{-m}, c q^{-m}, d q^{-m}, e q^{-m}, f ; q\right)_{k}}{\left(a q^{1-m} / b, a q^{1-m} / c, a q^{1-m} / d, a q^{1-m} / e, a q^{1-2 m} / f ; q\right)_{k}}\left(\frac{q^{2} a^{2}}{b c d e f}\right)^{k} \\
& \stackrel{(4.1)}{=} \frac{\left(a q^{-m}, f q^{m}, q a^{\frac{1}{2}},-q a^{\frac{1}{2}}, b, c, d, e ; q\right)_{-m}}{\left(a q^{1-m} / f, q^{1+m}, a^{\frac{1}{2}},-a^{\frac{1}{2}}, a q / b, a q / c, a q / d, a q / e ; q\right)_{-m}}\left(\frac{q^{2} a^{2}}{b c d e f}\right)^{-m} \\
& \times \frac{\left(a q^{1-2 m}, a q / d e, a q^{1-m} / d f, a q^{1-m} / e f, e q / c, f q^{1+m} / c, b q^{m} / a, b e f / a ; q\right)_{\infty}}{\left(a q^{1-m} / d, a q^{1-m} / e, a q^{1-2 m} / f, a q / d e f, q^{1+m} / c, e f q / c, b e / a, b f q^{m} / a ; q\right)_{\infty}} \\
& \times{ }_{8} \phi_{7}\left[\begin{array}{l}
e f / c, q(e f / c)^{\frac{1}{2}},-q(e f / c)^{\frac{1}{2}}, a q / b c, a q / c d, e f q^{m} / a, e q^{-m}, f \\
(e f / c)^{\frac{1}{2}},-(e f / c)^{\frac{1}{2}}, b e f / a, d e f / a, a q^{1-m} / c, f q^{1+m} / c, e q / c
\end{array} ; q, \frac{b d}{a}\right] \\
& +\frac{\left(a q^{-m}, f q^{m}, q a^{\frac{1}{2}},-q a^{\frac{1}{2}}, b, c, d, e ; q\right)_{-m}}{\left(a q^{1-m} / f, q^{1+m}, a^{\frac{1}{2}},-a^{\frac{1}{2}}, a q / b, a q / c, a q / d, a q / e ; q\right)_{-m}}\left(\frac{q^{2} a^{2}}{b c d e f}\right)^{-m} \\
& \times \frac{b q^{m}}{a} \frac{\left(a q^{1-2 m}, b q^{1+m} / a, b q / c, b q / d, b q / e, b q^{1-m} / f, d q^{-m} ; q\right)_{\infty}}{\left(a q^{1-m} / b, a q^{1-m} / c, a q^{1-m} / d, a q^{1-m} / e, a q^{1-2 m} / f, b d / a, b e / a ; q\right)_{\infty}} \\
& \times \frac{\left(e q^{-m}, f, a q / b c, b d e f q^{m} / a^{2}, a^{2} q^{1-m} / b \operatorname{def} ; q\right)_{\infty}}{\left(b f q^{m} / a, d e f / a, a q / \operatorname{def}, q^{1+m} / c, b^{2} q / a ; q\right)_{\infty}} \\
& \times{ }_{8} \phi_{7}\left[\begin{array}{l}
b^{2} / a, q b a^{-\frac{1}{2}},-q b a^{-\frac{1}{2}}, b q^{-m}, b c / a, b d / a, b e / a, b f q^{m} / a \\
b a^{-\frac{1}{2}},-b a^{-\frac{1}{2}}, b q^{1+m} / a, b q / c, b q / d, b q / e, b q^{1-m} / f
\end{array} ; q, \frac{a^{2} q^{2}}{b c d e f}\right],
\end{aligned}
$$

which equals the right-hand side of (4.2).

The above proposition can be viewed as a semi-finite form of Bailey's ${ }_{6} \psi_{6}$ summation formula. By taking $f=b$ and $m \rightarrow \infty$ in (4.2) while assuming $\left|a^{2} q / b c d e\right|<1$, we get

$$
\begin{aligned}
{ }_{6} \psi_{6}\left[\begin{array}{c}
q a^{\frac{1}{2}},-q a^{\frac{1}{2}}, b, c, d, e \\
a^{\frac{1}{2}},-a^{\frac{1}{2}}, a q / b, a q / c, a q / d, a q / e
\end{array} ;, \frac{a^{2} q}{b c d e}\right] \\
=\frac{(q / a, b d / a, a q / b c, a q / c d, a q, a q / d e, a q / b d, a q / b e ; q)_{\infty}}{(q / b, q / c, q / d, a q / b, a q / d, a q / e, a q / b d e, b d e / a ; q)_{\infty}} \\
\times \sum_{k=-\infty}^{\infty} \frac{(e ; q)_{k}}{(a q / c ; q)_{k}}(b d / a)^{k} \\
\stackrel{(2.2)}{=} \frac{(q / a, b d / a, a q / b c, a q / c d, a q, a q / d e, a q / b d, a q / b e ; q)_{\infty}}{(q / b, q / c, q / d, a q / b, a q / d, a q / e, a q / b d e, b d e / a ; q)_{\infty}} \\
\times \frac{(q, a q / c e, b d e / a, a q / b d e ; q)_{\infty}}{\left(a q / c, q / e, b d / a, a^{2} q / b c d e ; q\right)_{\infty}}
\end{aligned}
$$




$$
=\frac{(a q, a q / b c, a q / b d, a q / b e, a q / c e, a q / c d, a q / d e, q, q / a ; q)_{\infty}}{\left(a q / b, a q / c, a q / d, a q / e, q / b, q / c, q / d, q / e, q a^{2} / b c d e ; q\right)_{\infty}}
$$

Many proofs of the above identity have been found; see, for example, Slater and Lakin [11, Andrews [1, Askey and Ismail [2], Askey [3], Chen and Liu [5, Schlosser [9] and Jouhet and Schlosser [8]. Our proof shows that the semi-finite form of the ${ }_{6} \psi_{6}$ summation is in essence a shifted version of the ${ }_{8} \phi_{7}$ summation. This proof utilizes Ramanujan's ${ }_{1} \psi_{1}$ summation (2.2). It would be interesting to find a proof using a semi-finite (or even finite) form which yields Bailey's ${ }_{6} \psi_{6}$ summation in a direct limit, without the need to invoke another summation formula as above.

\section{ACKNOWLEDGEMENT}

We thank R. Askey, Wenchang Chu and M. E. H. Ismail for their valuable comments. In particular, we are indebted to M. Schlosser for crucial suggestions leading to considerable improvement of an earlier version. This work was done under the auspices of the 973 Project on Mathematical Mechanization, the Ministry of Education, the Ministry of Science and Technology, and the National Science Foundation of China.

\section{REFERENCES}

1. G. E. Andrews, Applications of basic hypergeometric functions, SIAM, Rev. 16 (1974), 441484. MR0352557 (50:5044)

2. R. Askey and M. E. H. Ismail, The very well poised ${ }_{6} \psi_{6}$, Proc. Amer. Math. Soc. 77 (1979), 218-222. MR0542088 (80m:33002)

3. R. Askey, The very well poised ${ }_{6} \psi_{6}$ II, Proc. Amer. Math. Soc. 90 (1984), 575-579. MR0733409 (85h:33001)

4. W. N. Bailey, Series of hyerpergeometric type which are infinite in both directions, Quart. J. Math. 7 (1936), 105-115.

5. W. Y. C. Chen and Z. G. Liu, Parameter augmentation for basic hypergeometric series, I, In: Mathematical Essays in Honor of Gian-Carlo Rota, Eds., B. E. Sagan and R. P. Stanley, Birkhäuser, Boston, 1998, pp. 111-129. MR1627355 (99f:33020)

6. G. Gasper and M. Rahman, Basic Hypergeometric Series, $2^{\text {nd }}$ ed., Encyclopedia of Mathematics and Its Applications, Vol. 96, Cambridge University Press, Cambridge, 2004. MR 2128719

7. M. E. H. Ismail, A simple proof of Ramanujan's ${ }_{1} \psi_{1}$ sum, Proc. Amer. Math. Soc. 63 (1977), 185-186. MR0508183 (58:22695)

8. F. Jouhet and M. Schlosser, Another proof of Bailey's ${ }_{6} \psi_{6}$ summation, Aequationes Math. 70 (2005), 43-50.

9. M. Schlosser, A simple proof of Bailey's very-well-poised ${ }_{6} \psi_{6}$ summation, Proc. Amer. Math. Soc. 130 (2002), 1113-1123. MR1873786 (2003b:33027)

10. M. Schlosser, Abel-Rothe type generalizations of Jacobi's triple product identity, in "Theory and Applications of Special Functions, A Volume Dedicated to Mizan Rahman" (M. E. H. Ismail and E. Koelink, eds.), Dev. Math. 13, Springer, New York, 2005, pp. 383-400. MR:2132472

11. L. J. Slater and A. Lakin, Two proofs of the ${ }_{6} \psi_{6}$ summation theorem, Proc. Edin. Math. Soc. 9 (1956), 116-121. MR0084600 (18:888b)

Center for Combinatorics, LPMC, Nankai University, Tianjin 300071, People's RePUBLIC OF CHINA

E-mail address: chen@nankai.edu.cn

Center for Combinatorics, LPMC, Nankai University, Tianjin 300071, People's RePUBLIC OF CHINA

E-mail address: fu@nankai.edu.cn 